\title{
Atomistic to Continuum Modeling of Solidification Microstructures
}

\author{
Alain Karma* \\ Department of Physics and Center for Interdisciplinary Research on Complex Systems, Northeastern University, Boston, MA 02115, USA
}

Damien Tourret

Los Alamos National Laboratory, Materials Science and Technology Division (MST 6), Los Alamos, NM 87545, USA

\begin{abstract}
We summarize recent advances in modeling of solidification microstructures using computational methods that bridge atomistic to continuum scales. We first discuss progress in atomistic modeling of equilibrium and non-equilibrium solid-liquid interface properties influencing microstructure formation, as well as interface coalescence phenomena influencing the late stages of solidification. The latter is relevant in the context of hot tearing reviewed in the article by M. Rappaz in this issue. We then discuss progress to model microstructures on a continuum scale using phase-field methods. We focus on selected examples in which modeling of $3 \mathrm{D}$ cellular and dendritic microstructures has been directly linked to experimental observations. Finally, we discuss a recently introduced coarse-grained dendritic needle network approach to simulate the formation of well-developed dendritic microstructures. This approach reliably bridges the well-separated scales traditionally simulated by phase-field and grain structure models, hence opening new avenues for quantitative modeling of complex intra- and inter-grain dynamical interactions on a grain scale.
\end{abstract}

Keywords: Multiscale solidification modeling, Atomistics, Interface pattern, Grain structure

\section{Introduction}

The quantitative prediction of solidification microstructures has been a long standing computational challenge $[15,5]$. It generally requires accurate modeling of the complex dynamics of the solid-liquid interface during the entire solidification process. The past two decades have witnessed major progress in modeling this dynamics through atomistic- and continuumscale simulations, and a handshake between those methods.

On the one hand, atomistic simulations have yielded accurate predictions of fundamental anisotropic properties of the solid-liquid interface for various metallic systems, such as the interface free-energy $[65,66,5,48]$ and kinetic coefficient $[69,66,130,53,101]$ that affect microstructural pattern formation over a wide range of solidification conditions. Those simulations typically use a million atoms and interatomic potentials modeled by the embedded-atom method (EAM). They have also yielded useful quantitative insights into solute-trapping in rapidly solidified alloys $[158,70]$, as well as interface coalescence phenomena that control crystal cohesion during the late stages of solidification $[67,46,111]$ and are relevant to hot tearing, reviewed in the article by Rappaz in this issue [118].

On the other hand, phase-field simulations have been extensively used to model the formation of monophase and polyphase solidification microstructures in a wide range of dendritic, eutectic and peritectic alloys $[16,5]$. Progress has been

\footnotetext{
* Corresponding author

Email addresses: a.karma@neu .edu (Alain Karma), dtourret@lanl.gov (Damien Tourret)
}

made to formulate quantitative phase-field models of alloy solidification for a wider range of phase diagrams [113] and diffusive transport properties $[19,20]$ within the theoretical framework of the thin interface limit $[79,38,77]$. Results of atomistic modeling have been incorporated into phase-field simulations to model complex observations. For example, the quantitative characterization of interface free-energy anisotropy derived from atomistic simulations has been useful to model changes of dendrite growth orientation [63, 35]. Moreover, boosted by steady increases in computing speed and massively parallel code implementations on multicore architectures such as graphic processing units (GPUs), phase-field simulations have become increasingly $3 \mathrm{D}$ and able to access experimentally relevant length and time scales $[124,12]$.

In the following section, we review recent advances in atomistic modeling of solid-liquid interface properties and coalescence, focusing on the prediction of key parameters influencing microstructure formation or hot tearing on a larger scale. In section 3, the core section of this article, we review progress in phase-field modeling focusing on cellular and dendritic microstructures. We leave out two-phase microstructures, reviewed in the separate article by Plapp in this issue [114]. In section 4 , we discuss recent progress to bridge the gap between phase-field modeling on the microstructure scale and grain structure modeling [118]. This gap stems from the fact that, while $3 \mathrm{D}$ volumes up to about $\mathrm{a} \mathrm{mm}^{3}$ are within reach of today's large scale phase-field computations, those volumes are still minute on the scale of a casting. In contrast, Cellular Automata coupled with Finite Elements (CAFE) mod- 
els $[119,51,118]$ can access those much larger scales and predict grain structures of castings [26]. However, those models do not resolve dynamical interactions between branches of hierarchical dendritic networks, which can strongly influence both intra-grain microstructure selection and the growth competition of different grains [139]. Section 4 describes a dendritic needle network (DNN) approach designed to quantitatively model those interactions on the grain scale, beyond the current reach of phase-field modeling. While this approach is not a substitute for grain structure models, it opens new avenues to test and improve those models by investigating complex branch interactions that shape the grain structure [138].

\section{Atomistic modeling}

\subsection{Solid-liquid interface properties}

The advent of microscopic solvability theory during the 1980 's $[86,81]$ led to the understanding that anisotropic properties of the solid-liquid interface have a profound influence on dendrite growth. Those properties generally determine the selection of both the dendrite tip operating state and growth direction. Predictions of solvability theory were validated by phasefield simulations during the 1990's [78, 79] and the first decade of this millennium witnessed major progress in characterizing interfacial properties by atomistic modeling $[65,66,5]$. Those studies yielded the novel insight that at least two parameters $a_{1}$ and $a_{2}$ (also commonly denoted by $\epsilon_{1}$ and $\epsilon_{2}$ [66]) are necessary to describe the anisotropy of the solid-liquid interfacial free-energy $\gamma_{s l}$, as opposed to a single parameter $a_{1}$ previously considered [86, 81, 78, 79]. Since this anisotropy is weak in metallic systems forming fcc or bcc crystal structures, the variation of $\gamma_{s l}$ with the direction normal to the interface, $\hat{n}$, can be parameterized by an expansion in Kubic harmonics

$$
\gamma_{s l}(\hat{n})=\gamma_{0}\left[1+a_{1} K_{1}(\hat{n})+a_{2} K_{2}(\hat{n})+\cdots\right]
$$

where the $K_{i}$ 's are combinations of spherical harmonics with cubic symmetry. The first harmonic $K_{1}(\hat{n})$ favors dendrite growth along $\langle 100\rangle$ directions for $a_{1}>0$, while the second $K_{2}(\hat{n})$ favors $\langle 110\rangle$ directions for $a_{2}<0$. Molecular dynamics (MD) simulations of several fcc metals (e.g. $\mathrm{Cu}, \mathrm{Ni}, \mathrm{Al}$, $\mathrm{Au}, \mathrm{Ag}$ ) and alloys (e.g. $\mathrm{Ni}-\mathrm{Cu})$ reviewed in [5] have predicted that $a_{1}>0$ and $a_{2}<0$ corresponding to the ordering $\gamma_{s l}^{100}>\gamma_{s l}^{110}>\gamma_{s l}^{111}$; the magnitude of $\gamma_{s l}$ was reasonably well predicted by the Turnbull relation [143] $\gamma_{s l}=\alpha L Q^{2 / 3}$ with a Turnbull coefficient $\alpha \approx 0.55$ for fcc ( $\mathrm{L}$ is the latent heat of melting per atom and $\varrho$ is the solid atomic density). A study of a model alloy system with nearly ideal solution thermodynamics has also predicted that alloying tends to make $a_{2}$ more negative, thereby potentially promoting growth towards $\langle 110\rangle$ [10]. This knowledge has motivated detailed phase-field modeling and experimental studies of changes of dendrite growth directions in Al-Zn alloys, discussed in the next section. Those changes are explained by the assumption that $\mathrm{Zn}$ addition changes the $\gamma_{s l}$ anisotropy so as to induce a transition from $\langle 100\rangle$ to $\langle 110\rangle$ directions, but atomistic modeling remains needed to predict $a_{1}$ and $a_{2}$ for this alloy system to validate this scenario.
While dendrite growth is controlled by $\gamma_{s l}$ and its anisotropy for low solidification rate, it becomes strongly influenced by anisotropic interface kinetics for large solidification rates [22, 72, 21]. MD simulations have been used to compute the interface kinetic coefficient $\mu$ (proportionality constant between interface velocity and undercooling) and its anisotropy for various pure metals $[68,69,66,130,53,101]$. Simulations for fcc (e.g. Ni) and bcc (e.g. $\mathrm{Fe})$ have predicted the ordering $\mu^{100}>\mu^{110}>\mu^{111}$ that favors dendrite growth along $\langle 100\rangle \mathrm{di}-$ rections, as commonly, albeit not uniquely, observed in deeply undercooled melt experiments. This ordering was predicted by a theoretical study of bcc crystallization kinetics that exploits a Ginzburg-Landau (GL) expansion of the excess solid-liquid free-energy [156]. There, the GL expansion was expressed in terms of the amplitudes of density waves corresponding to principal reciprocal lattice vectors of the crystal lattice. A similar expansion had been used to predict $\gamma_{s l}$ and its anisotropy [155] in equilibrium. In its extension outside of equilibrium, GL theory is closely related to the linear density wave theory of $\mu$ by Mikheev and Chernov (MC) [97, 30]. The anisotropy of $\mu$ arises naturally in both theories from the dependence of density wave profiles across the solid-liquid interface on the interface normal direction $\hat{n}$. Both theories also predict that $\mu$ is inversely proportional to the relaxation time $\tau_{L}$ of density waves in the liquid, itself related to the liquid diffusivity. Using a direct computation of $\tau_{L}$ by MD simulations for an EAM model of bcc Fe [156], GL theory was found to predict much more accurately than the MC theory the magnitude of $\mu$ and its anisotropy obtained in free-solidification MD simulations for the same EAM potential [53]. This improvement can be attributed to the fully nonlinear description of density wave profiles across the solidliquid interface in GL theory. The prediction that $\mu \sim 1 / \tau_{L}$ is also consistent with free-solidification MD simulations showing that, for different EAM potentials and small interface undercooling, $\mu$ correlates well with the liquid diffusivity [96].

In addition to determining $\mu$ in pure metals, MD simulations have also probed the strong departure from chemical equilibrium at the solid-liquid interface during rapid alloy solidification for both a Lennard-Jones binary system and an EAM model of Ni-Cu $[158,70]$. The results have confirmed the expected transition to complete solute trapping at high growth rates predicted by different analytical models [7, 50, 75], and demonstrated the existence of solute drag previously examined using both sharp-interface [7] and phase-field models [2]. They have also shown solute drag to be anisotropic [158], potentially affecting both dendrite growth rate and orientation [70].

\subsection{Interface coalescence}

While crystal cohesion is energetically favored when solidliquid interfaces from the same grain impinge upon each other during the late stages of solidification, it can become energetically disfavored when the two grains are sufficiently disoriented. If the grain-boundary (GB) free-energy $\gamma_{g b}$ exceeds twice $\gamma_{s l}$, i.e. $\Delta \gamma \equiv \gamma_{g b}-2 \gamma_{s l}>0$, the formation of a thin intergranular liquid-like film becomes energetically favored at temperatures below the melting point. This GB premelting phenomenon has been widely studied experimentally [28, 8, 71, 92, 
$145,152,36,73,37,91,59]$ and theoretically using lattice models [82], phase-field models [89, 134, 135, 99, 151, 121], MD simulations [24, 146, 154, 67, 46, 111], and phase-field-crystal simulations that resolves the atomic-scale crystal density field $[14,95,1]$. In a solidification context, intergranular films delay crystal cohesion, which occurs at a bridging temperature below the liquidus temperature when $\Delta \gamma>0$, thereby reducing shear resistance. For this reason, $\Delta \gamma$ is a key parameter in continuum models of hot tearing [120, 150, 5] reviewed in Ref. [118].

Atomistic modeling has been used to characterize quantitatively the structural forces that control the intergranular layer width $w[95,67,46,1]$. This characterization has been carried out by defining a disjoining potential $\Psi(w)$ via the excess Gibbs free-energy per unit of GB area

$$
G_{\mathrm{exc}}(w, T)=\Delta G(T) w+\Psi(w)
$$

where $\Delta G(T)=G_{S}(T)-G_{L}(T)$ is the bulk Gibbs free-energy difference between solid and liquid and $\Psi(w)-2 \gamma_{s l}$ represents the part of this excess Gibbs free-energy due to the overlap of spatially diffuse solid-liquid interfaces from the two impinging grains. Minimization of $G_{\text {exc }}(w, T)$ with respect to $w$ using the simple analytical form $\Psi(w)=2 \gamma_{s l}+\Delta \gamma \exp (-w / \delta)[153,120]$, where $\delta$ is proportional to the solid-liquid interface width, predicts that $w$ diverges at the melting point $T_{m}$ for $\Delta \gamma>0$ and remains zero up to a temperature exceeding $T_{m}$ for $\Delta \gamma<0$.

MD simulations that exploit fluctuations of layer width to compute $\Psi(w)[67,46]$ have shown that high-energy GBs exhibit a purely repulsive disjoining potential that is consistent with the exponential form of $\Psi(w)$ for $\Delta \gamma>0$, as illustrated in Fig. 1 for a pure Ni twist GB. However, for lower-energy GBs, $\Psi(w)$ exhibits a shallow minimum that corresponds to a finite layer width at the melting point, as illustrated for a pure Ni tilt GB in Fig. 1. This nanometer-scale width represents a compromise between attractive and repulsive parts of the disjoining potential that are both structural in nature, since they are mediated by the overlap of the tails of the crystal density fields and crystal-defect formation energy, respectively. The disjoining potential $\Psi(w)$ also generally contains an attractive part due to London dispersion forces that are not accounted for in MD or PFC simulations, but play an important role in other systems (e.g. ceramic materials [32]). However, in metallic systems, dispersion forces can be estimated to yield a contribution to $\Psi(w)$ on the order of $\mathrm{mJ} / \mathrm{m}^{2}$ that is negligible on the scale of Fig. 1. Similar GB premelting behaviors are also predicted by PFC simulations [95, 1], which have been compared quantitatively to MD simulations for classical models of pure Fe [1]. PFC [95] and MD [111] studies have also yielded the insight that the condition $\Delta \gamma=\gamma_{g b}-2 \gamma_{s l}>0$ can only accurately predict GB premelting if one takes into account the fact that $\gamma_{g b}$ is significantly reduced at the melting point (compared to zero temperature) due to the elastic softening of the material. Those studies have also revealed the existence of novel transitions between different GB structures mediated by pairing of dislocations [95, 111].

Atomistic modeling has also been used recently to characterize the response of high-temperature GBs to a shear stress
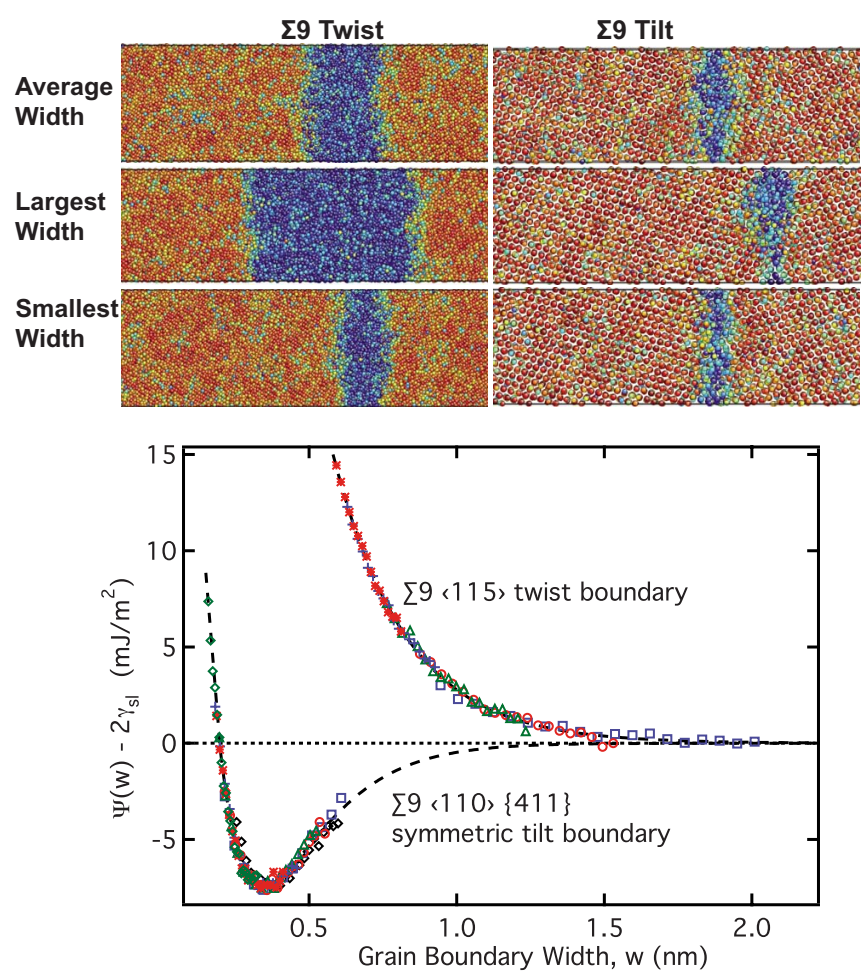

Figure 1: Snapshots of MD simulations of pure Ni modeled with an EAM potential at an undercooling of $2 \mathrm{~K}$ below the melting point with solid (liquid) atoms colored in red (blue). Snapshots on the top left illustrate different configurations for a wet $\Sigma 9$ twist boundary that exhibits large fluctuations of the liquid-layer width $w$ [67], while those on the top right are for a dry $\Sigma 9$ tilt boundary that exhibits smaller width fluctuations [46]. The bottom figure shows the disjoining potentials for those two boundaries. The twist boundary exhibits a purely repulsive potential, corresponding to a diverging liquid layer width at the melting point, while the potential for the tilt boundary exhibits a minimum corresponding to a finite width at the melting point.

$[25,74,142,141,80]$. An exhaustive MD study of [100] symmetric tilt GBs in pure $\mathrm{Ni}$ [25] has shown that the range of GB misorientation where GB sliding is observed in response to a shear stress increases close to the melting point, consistent with the view that the formation of an intergranular liquid-like film favors sliding. However, a recent combined PFC and MD study [141] also showed that asymmetrical GBs in fcc metals can exhibit sliding unrelated to premelting. While those results have not been incorporated to date in mesoscale models of mechanical behavior of the mushy zone and hot tearing, they should be relevant for understanding those complex phenomena. Finally, while multiphase-field modeling has been used to investigate interface coalescence in simple binary alloys [151], MD studies remain limited to pure metals. Those studies still need to be extended to metallic alloys to understand more quantitatively the influence of solute addition on hot cracking sensitivity.

\section{Phase-field modeling}

\subsection{Quantitative phase-field modeling}

Due to their simplicity and potential in handling complex free-boundary problems, phase-field (PF) models have become 
ubiquitous in materials science [16, 29]. They are well adapted to study solid-liquid interface dynamics during solidification. Quantitative predictions have long been limited by the requirement of a diffuse interface width of comparable size to the physical interface, i.e. a few atomic planes. However, the development of asymptotic analysis of PF equations, now referred to as the thin interface limit, has made it possible to use a numerical interface thickness several orders of magnitude larger than the physical interface thickness [79, 77, 38]. For alloy solidification, a quantitative PF formulation was originally formulated for isothermal [77] and non-isothermal [38] solidification by introducing an anti-trapping current [77, 38]. This current compensates for the excess solute trapping associated with a mesoscale interface thickness and yields the desired thin-interface limit for low solidification rate, where the interface can be assumed to be in local thermodynamic equilibrium. However, this formulation is only valid for dilute alloys and vanishing solute diffusivity in the solid. Recent progress has been made to develop quantitative PF models of binary alloy solidification that also utilize an anti-trapping current, but remain valid for arbitrary phase diagrams [113] or for a finite solid diffusivity $[23,19,20]$. The generalization to arbitrary phase diagram is based on deriving the PF equations variationally from a grand-potential, instead of a free-energy, functional, and concomitantly using the chemical potential as dynamical variable instead of the solute concentration [113]. This approach also unifies different physical interpretations of alloy PF models (see [113] and earlier references therein). The extension to finite solid diffusivity, in turn, exploits additional freedom in model formulation by introducing a cross coupling between the nonconserved phase field and the conserved concentration field within the formal theoretical framework of irreversible thermodynamics and the Onsager relations [23, 19, 20].

\subsection{Computationally intensive phase-field implementations}

A major contribution to bridging scales with phase-field modeling has been the fast growth of computational capabilities. Numerous approaches have been proposed to take advantage of the available computational power including adaptive mesh refinement [116], optimized implementations on parallel architectures [124], hybrid finite-difference and random-walk algorithms [115], implicit time stepping schemes and multi grid approaches [58, 17], and methods that exploit up-scaling techniques and symmetry [13]. We highlight two of these approaches that yielded significant insight into microstructure selection, namely mesh refinement and massive parallelization facilitated by the growing use of Graphic Processing Units (GPUs).

Phase-field remains the method of choice for simulating complex interface patterns in solidification. However, a quantitative prediction of growth dynamics with PF requires an accurate description of the dendrite tip curvature. This limit may be circumvented using a non-uniform discretization of the simulation domain, with an increased accuracy, i.e. a finer mesh, where it matters the most, in the present case close to the solid-liquid interface and the dendritic tips. Dynamic (adaptive) remeshing techniques for PF calculations [117] — also applicable to atomistic phase-field-crystal calculations [6] — allow efficient simulations up to the scale of an entire dendritic array. Resulting simulations have shed light on several microstructure selection mechanisms [3, 61, 4, 109, 60, 102], and have been coupled to larger-scale heat transfer models and probabilistic nucleation models to predict microstructure distribution in processes such as laser powder deposition [44] and welding [103].

From a hardware perspective, the parallel architecture of GPUs, first developed for graphics in video games and movies, has recently emerged as a powerful and cost-effective tool for intensive computations. With the development of GPUs dedicated to high performance computing, these massively parallel architectures - e.g. 2,880 cores in one Nvidia ${ }^{\circledR}$ Tesla ${ }^{\circledR}$ K40 - have been increasingly used in scientific computing. The most advanced use of GPUs for solidification simulation is the work of Aoki, Takaki, Yamanaka, and co-workers [157, 124], reaching the petaFLOPS calculation on the Japanese TSUBAME 2.0 supercomputer, using a hybrid CPU-GPU algorithm for a three-dimensional competitive grain growth simulation - 4,000 GPUs along with 16,000 CPU cores, for $4,096 \times 6,500 \times 10,400$ grid points [124]. While such intensive calculations require a significant investment in terms of implementation, the simplest single GPU implementations already allow 3D simulations that are two orders of magnitude faster than using a single CPU core $[157,139]$.

Alongside with adaptive remeshing, parallelization techniques on GPUs or other archictectures - e.g. using MPI [54, 106] or OpenMP [106, 56] - allow simulations at experimentally relevant length/time scales [12, 56, 139]. The following subsection highlights a few examples of how the combination of theoretical approaches and advanced computational techniques can lead to a deeper understanding of growth dynamics and microstructure selection in solidification processing.

\subsection{Selected applications}

\subsubsection{Comparison to directional solidification experiments}

The thin interface approach, extended to non-isothermal solidification and combined with the anti-trapping current, has shown quantitative agreement with directional solidification experiments [38]. It has provided an exact benchmark for analytical models of tip undercooling selection, and highlighted complex mechanisms such as the existence of stability gaps in pattern selection at low interfacial anisotropies $[62,12]$. The longstanding problem of dendritic sidebranching (SB) origin was confirmed to be noise amplification [86], while a bifurcation to a limit-cycle oscillatory branch of deterministic SB was shown to exist at large spacings [39]. Interestingly, a higher thermal gradient was found to reduce the onset velocity and wavelength of $\mathrm{SB}$, due to the dendrite tip becoming blunter, thus promoting noise amplification.

This PF model, implemented on GPUs, yields quantitative predictions on complex nonlinear dynamics of spatially extended solid-liquid interfaces. This is exemplified in Fig. 2 by a recent comparison of $3 \mathrm{D}$ simulations to directional solidification experiments with in situ imaging of the dynamics 

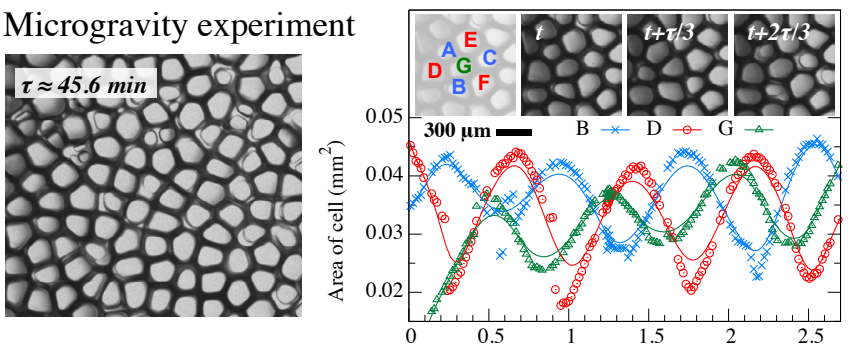

Phase-field simulation
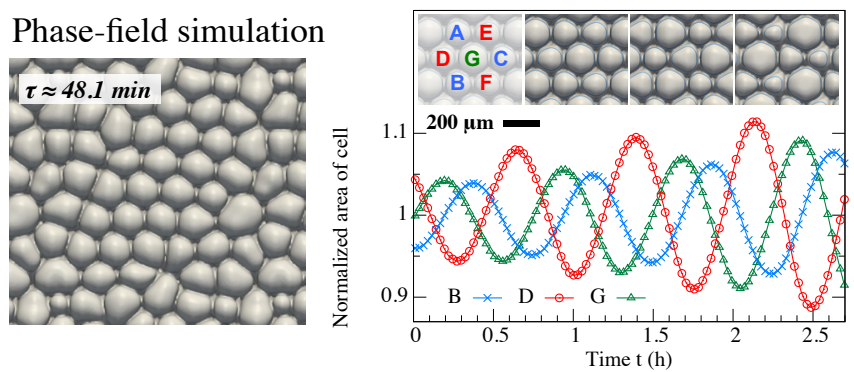

Figure 2: Oscillations in three-dimensional cellular patterns [12]. Locally ordered regions of the array exhibit coherent breathing-mode oscillations. Microgravity experiments (top) and phase-field simulations (bottom) are in excellent quantitative agreement. In both, cells belonging to three sublattices of the hexagonal array oscillate $2 \pi / 3$ out-of-phase with a period $\tau$ of 45 minutes.

of spatially extended cellular arrays under microgravity conditions where fluid flow is suppressed [12]. There, PF simulations on time scales of several hours, reproduce oscillatory breathing modes observed in cellular arrays with a striking agreement on the oscillation period. The simulations have also shown that the absence of long-range spatial coherence of those oscillations is due to tip splitting events that maintain a disordered cellular array structure, unlike in two-dimensions where coherent oscillations are observed [55].

\subsubsection{Orientation transition and twinning in Al-Zn alloys}

Atomistic simulations, reviewed in section 2, have shown that alloying may give rise to substantial changes in interface free-energy anisotropies [10]. Phase-field simulations of equiaxed dendritic growth have revealed the existence of a continuous transition of growth direction from $\langle 100\rangle$ to $\langle 110\rangle$ as a function of anisotropy parameters [63]. A qualitatively similar transition was observed to occur as a function of increasing $\mathrm{Zn}$ concentration in the fcc $\mathrm{Al}$ phase in directionally solidified Al-Zn alloys [63], suggesting that anisotropy parameters are changing as a function of $\mathrm{Zn}$ concentration. This transition has been further characterized both experimentally by directional solidification of Al-Zn alloys [47] and numerically [35] by phase-field simulations that model quantitatively those experiments. Those simulations, illustrated in Fig. 3, show a continuous transition of dendrite growth directions over a narrow range of anisotropy parameters, producing hyperbranched structures in the form of textured seaweeds [35].

Further investigations on microstructure selection in $\mathrm{Al}-\mathrm{Zn}$ alloys have focused on the growth morphology of twinned dendrites. Experimental [122] and phase-field [123] studies have suggested the presence of a groove at the tip of twinned den-

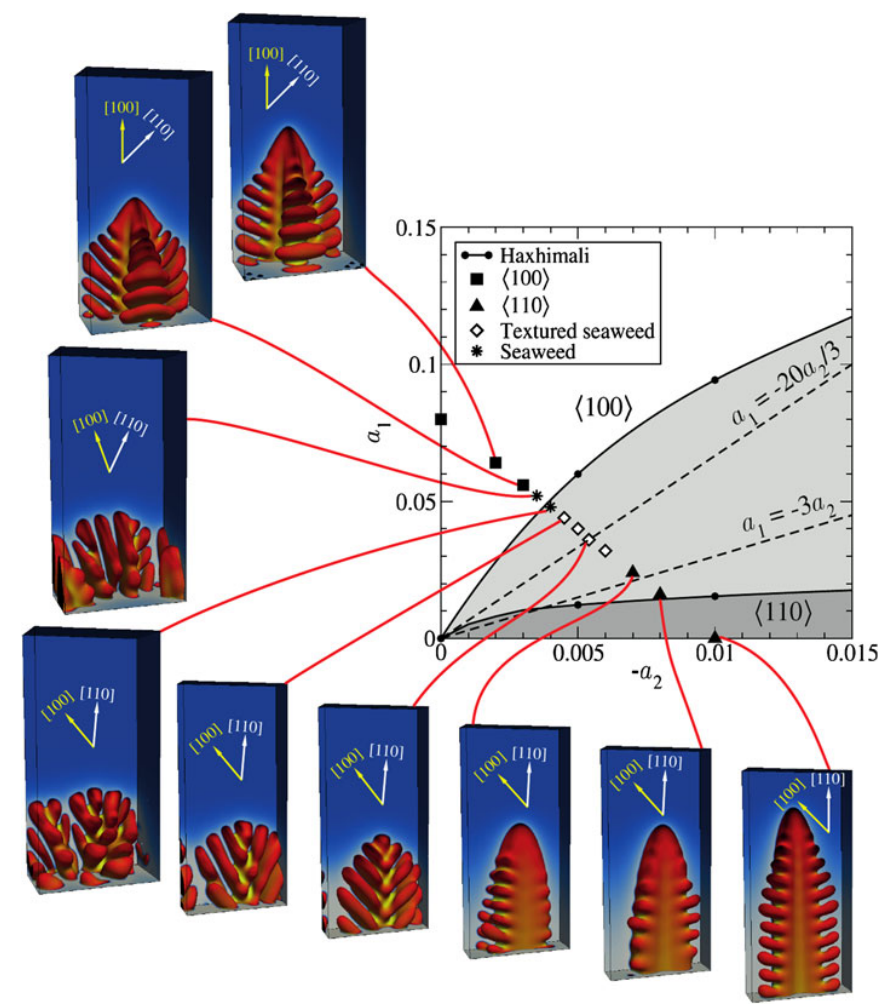

Figure 3: Phase-field prediction of the dendrite orientation transition from $\langle 100\rangle$ to $\langle 110\rangle$ in directional solidification of Al-Zn alloys, as a function of the two anisotropy parameters $a_{1}$ and $a_{2}$ defined in Eq. (1). [35]

drites, evolving into a doublon morphology, associated with the formation of small pockets of highly concentrated liquid at the doublon root for relatively high solidification rates. Recently, new experiments have brought strong evidence that twinned dendrites may nucleate on the faces of a fivefold symmetric phase, such as icosahedral quasicrystals or $\mathrm{Al}_{45} \mathrm{Cr}_{7}$. This nucleation mechanism provides a natural explanation for the significant increase in twinned dendrites and the subsequent grain refinement in $\mathrm{Al}-\mathrm{Zn}$ alloys when minute amounts of $\mathrm{Cr}$ are added [84]. A similar mechanism was reported in gold alloys when adding small amounts of Ir into the melt [85].

\subsubsection{Intragrain microstructure selection}

At the scale of a dendritic array, phase-field simulations have been shown to reproduce experimentally established scaling laws for the steady-state selection of primary spacing as a function of processing conditions and crystal orientation [139]. As the growth velocity is increased, PF simulations can predict the transition of the cell/dendrites growth direction from the orientation of the temperature gradient to the orientation of the crystal, as well as the morphology transition to degenerate structures at high temperature gradient and/or high crystal misorientation $[4,56,139]$. However, while two-dimensional simulations qualitatively reproduce realistic scaling laws, full threedimensional simulations remain necessary to reach a quantitative agreement with experiments [56].

Provatas and co-workers have also shown that twodimensional PF simulations can reproduce transient spacing 
evolution in directional solidification [3]. This evolution is characterized by plateau-like regions of constant primary spacing that are observed experimentally [90], which can be related to the hysteretic nature of the dynamical selection of the spacing as a function of pulling velocity [61]. Adding the effect of repulsive grain boundaries [109], they investigated grain boundary coalescence at high solid fraction, the formation of highly segregated liquid pools, and the evolution of solid connectivity during late stage solidification $[60,102]$.

\subsubsection{Intergrain microstructure selection}

Much attention was recently given to grain growth competition in polycrystalline directional solidification [40, 41, 87, 133, 132, 139]. A conceptual framework to understand this competition was originally proposed by Walton and Chalmers [147]. They assumed that a grain growing at a higher misorientation with respect to the temperature gradient direction cannot overgrow a better-oriented grain because the latter grows with a smaller undercooling and hence ahead of the misoriented grain. However, observations [164] and phase-field simulations [40, 41, 133] have suggested more complex mechanisms of grain-boundary evolution during solidification.

Using 2D phase-field, several groups have confirmed the occurrence of overgrowth of the better-oriented grain at the converging GB [87, 132, 139]. The underlying mechanism was shown to be diffusive interactions between dendrite tips. As misoriented dendrites impinge upon the well-oriented grain, the local spacing progressively decreases in the well-oriented grain close to the GB, and its local undercooling increases, until elimination of the dendrite closest to the GB [87, 132]. However, the frequency of this overgrowth mechanism at the converging GB is relatively low, such that the orientation of the converging GB remains close the temperature gradient orientation [139].

In contrast, grain competition at the diverging GB is controlled by the growth of tertiary branches and appears more complex [40, 41, 139]. Since dendritic sidebranches originate from selective amplification of noise, microscopic thermal fluctuations may have significant macroscopic consequences on the selection of GB orientation. This was evidenced by running series of 2D simulations of grain competition with similar control parameters, but different random number seeds in PF calculations [139]. Therefore, orientation selection of the diverging GB has to be regarded as a stochastic process, and treated statistically in order to obtain relevant quantitative data, which makes the task all the more computationally challenging.

With the exception of a few large scale 3D simulations [40, 41, 133], most recent systematic PF studies of columnar growth competition were achieved using 2D simulations with one grain aligned along the temperature gradient direction [87, 132, 139]. In this configuration, grain elimination is relatively slow [139]. Even in two dimensions, the combination of orientations of both crystals has a strong influence on GB orientation selection [40, 41], and a slight misorientation of the better-oriented grain can yield a much faster grain elimination, due to the preferential orientation of secondary sidebranches [136].

Furthermore, in order to reproduce realistic experiments, one needs to consider the 3D orientation of the two (or more) crys-
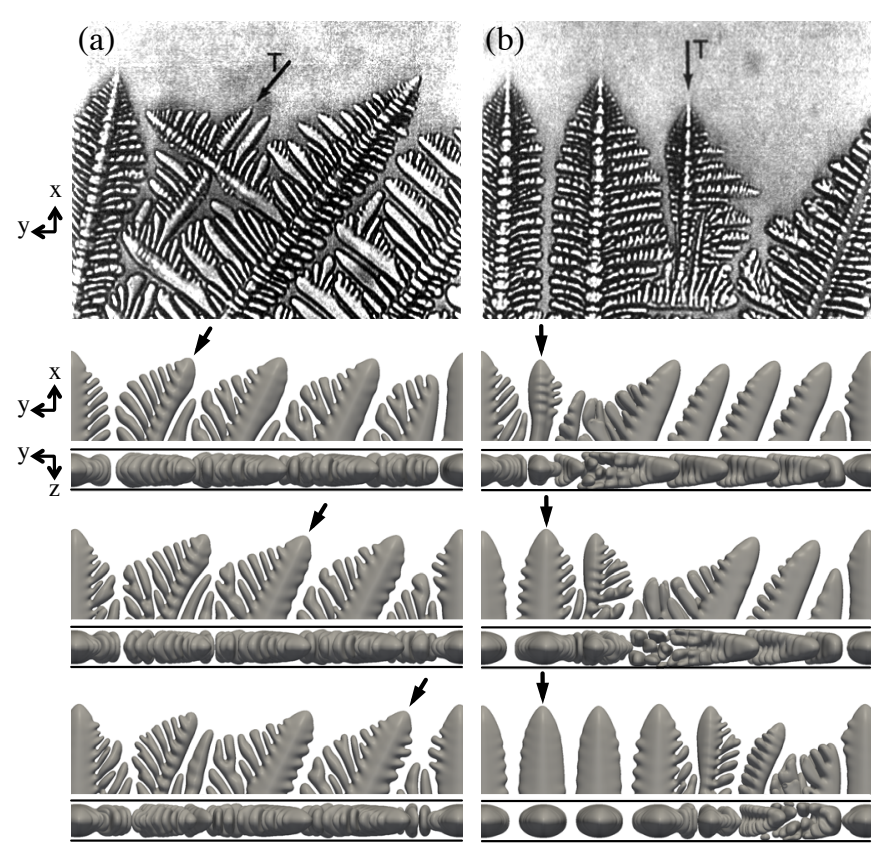

Figure 4: Dendritic grain growth competition in three-dimensional thin-sample directional solidification. Experimental pictures (top) are from [52]. The arrow in each phase-field simulation (bottom) follows a unique dendrite in time. [125]

tals. This is illustrated in Fig. 4 with 3D simulations reproducing the mechanisms exposed by Gandin et al. in thin sample experiments [52]. When the two grains have their crystalline axes parallel or normal to the thin sample walls in (a), tertiary branching occurs preferentially from the misoriented grain and grain elimination is limited. When the misoriented crystal exhibits a second angle with the walls, e.g. $45^{\circ}$ in (b), sidebranching from that grain is inhibited, tertiary branches originate from the best oriented grain, and grain elimination is much faster (see Fig. 12 in [139] for a schematic description).

\subsection{Other advances in phase-field modeling}

\subsubsection{Mixed transport}

Since the introduction of the no-slip condition at the solidliquid interface to model melt flow in phase-field simulations [11], several studies have shed light on microstructure selection mechanisms in the presence of convection. The solidliquid density change in free growth was shown to affect the dendritic tip selection parameter [131]. However, a reformulation accounting for density changes [43] provides a good estimation of the tip selection parameter independent of the relative density change. Two-dimensional simulations have shown that modulated flow with a given pulse duration can yield deterministic sidebranching events [108], much like with a short thermal perturbation of a dendrite tip in diffusive growth [39]. However, dimensionality plays a critical role in the study of convective flows around dendrites [160] and three-dimensional simulations appear mandatory to achieve conclusive studies on fluid flow effect on dendritic microstructure selection.

An alternative approach to fluid flow consists in replacing the full resolution of Navier-Stokes equations by Lattice 
Boltzmann (LB) equations. Whether using a solidification model based on LB equations [98, 129], coupling with phasefield models [93, 107], or with cellular-automaton based models [159], this simple and computationally efficient approach provides a powerful scale-bridging tool and may yield valuable insight into convective crystal growth in the future.

\subsubsection{Rapid solidification}

Rapid solidification is of particular interest in the context of microstructure selection in processes such as welding, alongside with the growing interest in additive manufacturing. A model recently introduced by Steinbach and co-workers replaces the interface equilibrium partitioning condition by coupling two concentration fields linked by a kinetic equation, describing the exchange of components between phases [128]. This approach introduces an effective interface permeability parameter, which can be adjusted by comparison to smaller scale thin-interface calculations for rapid solidification [49, 161]. This method has also been extended to multicomponent alloys [162]. A similar model, based on thermodynamic extremal principle was also recently proposed [149, 148]. Such approaches were found to successfully predict solute trapping effects experimentally measured in Si-As alloys [128, 149].

Additionally, using a coupled thermal-solutal thin-interface phase-field model, Mullis and co-workers have recently discussed the validity of the anti-trapping current in the context of rapid solidification, when solute trapping is expected to occur, and proposed strategies to adapt the strength of the corrective current to retrieve quantitative predictions [105]. They have also shown an evolution of the the tip selection parameter at high undercooling [104], and suggested it as a possible explanation for grain refinement in deeply undercooled melt [105, 104].

\subsubsection{Multi-phase and multicomponent systems}

In order to model industrially-revelant alloys and structures, one needs quantitative PF models for multi-grain, multi-phase, multicomponent systems [114].

For polycrystalline structures, the orientation-field PF model has been developed that uses only one additional scalar field corresponding to the grain orientation in 2D [83]. This model naturally describes grain rotation owing to the rotational invariance of the underlying free-energy and dynamical equations. A different formulation of this model was recently proposed in an attempt to minimize spurious long-range GB interactions associated with the method [64]. However, limitations of those models have been recently emphasized [112]. The multi-phasefield approach, with each grain attached to an individual phase field [127] remains a practical solution in common situations where grain rotation, which is not described by this approach, is absent. The method is more computationally demanding, and requires advanced algorithms to avoid a drastic increase in memory usage $[57,144]$. In addition, polycrystalline solidification can also be modeled with a single phase-field and an integer orientation field in situations, such as the growth competition of columnar grains [139], where the GB groove is situated deep into the mushy zone and need not be modeled accurately.
Several approaches have been proposed in recent years to handle quantitative PF simulations of multicomponent alloys solidification [42, 31, 100, 110, 162]. One approach exploits a grand-potential functional [31] that uses the chemical potential as dynamical variable instead of the solute concentration, similarly to the approach introduced for binary alloys [113]. This grand potential formulation, which has also been extended to rapid solidification [34], appears to be a promising candidate to handle alloys with arbitrary phase diagrams, with the potential to be coupled with CalPhaD calculations (see e.g. $[42,18,45,163])$.

\section{Towards the grain scale}

In concentrated alloys, solidification usually produces grains in the form of hierarchical networks of sharp needle-like branches, like in Fig. 5a. Thus, several orders of magnitude separate the scale of the tip radius $\rho$ and the diffusion length $D / V$ where $D$ is the solute diffusivity and $V$ the growth velocity. Such morphologies are challenging to model with PF simulations that need to resolve the solid-liquid interface of each branch. To overcome this limitation, we recently developed a multiscale Dendritic Needle Network (DNN) model [138, 140] that is briefly reviewed in this section.

The DNN model represents the hierarchical dendritic network (e.g. Fig. 5a) by its ensemble of branches approximated as thin needles that interact with each other through the longrange diffusion field (Fig. 5b) [76, 138]. The solid-liquid interface along each needle is assumed to be in local equilibrium.

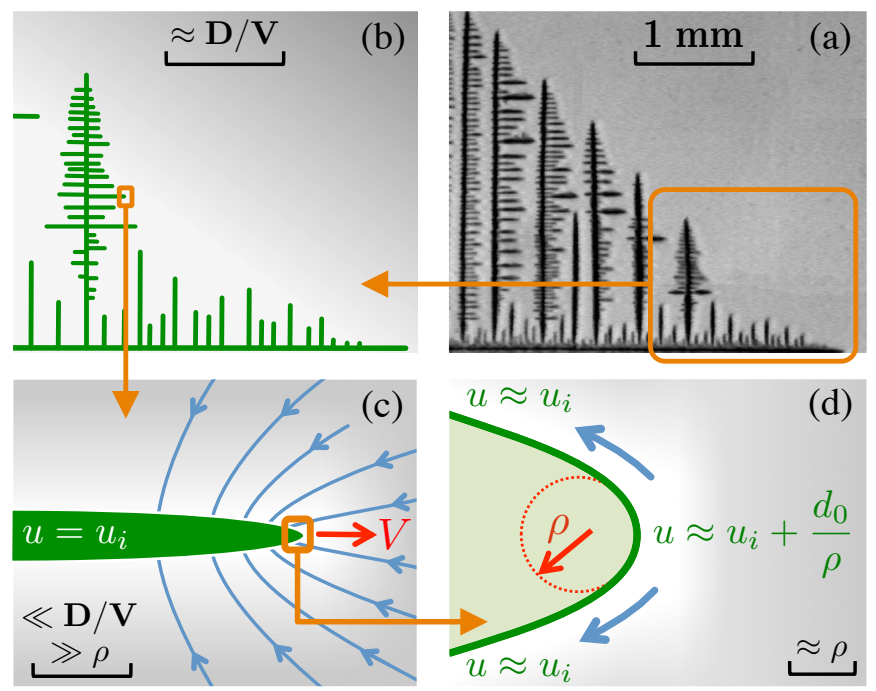

Figure 5: The multiscale Dendritic Needle Network (DNN) model represents a dendritic grain - such as the ammonium-bromide crystal in (a) from [33] - by a network of thin needle crystals that interact through the solute field, as illustrated in (b). The growth dynamics of each individual needle branch is obtained by combining two independent conditions at two distinct length scales: (c) a solute conservation equation at a length scale much larger than the needle tip radius $\rho$ but much smaller than the diffusion length $D / V$, and (d) a solvability condition at the scale of the needle tip radius $\rho$ [138]. The $u$ field is a dimensionless measure of the departure of the concentration field from its equilibrium value $u_{i}$ for a reference flat interface. 
The growth dynamics of each individual needle can then be uniquely prescribed by two independent conditions. The first condition stems from the fact that the concentration gradient normal to the solid-liquid interface is largest at the needle tip as depicted schematically in Fig. 5c. Based on the form of the diffusion field in the near-tip region on an intermediate scale much larger than $\rho$ but much smaller than $D / V$, it is possible to define a solutal flux intensity factor $\mathcal{F}(t)$ that represents the instantaneous strength of this gradient averaged over the tip region. $\mathcal{F}(t)$ can be calculated using a condition of solute conservation expressed as a contour (surface) integral around the tip in 2D (3D) [140], which yields the result that $\mathcal{F}(t)$ is proportional to the product $\rho V^{2}$ in 2D and $\rho V$ in 3D [138, 140]. The second condition is identical to the standard microscopic solvability condition that relates the product $\rho^{2} V$ (also the selection parameter $\sigma=2 D d_{0} / \rho^{2} V$, with $d_{0}$ the solute capillarity length at the tip) to the strength of the interfacial energy anisotropy [86, 9]. While this condition was originally derived for steady-state growth conditions [86, 9], subsequent PF simulations demonstrated that $\rho^{2} V$ remains approximately constant in time during transient growth conditions where both $\rho$ and $V$ are temporally varying [115]. This finding reflects the fact that the solvability condition that determines $\rho^{2} V$ is obtained by a solution to the interface growth equations on the tip scale (Fig. 5d), and hence should be expected to remain valid in situations where the diffusion field is slowly evolving on a scale much larger than $\rho$, as seen in PF simulations [115]. This key property allows us to assume that $\rho(t)^{2} V(t)$ remains constant in time in the DNN model and hence, together with the other condition $\mathcal{F}(t) \sim \rho(t) V^{2}(t)$ in $2 \mathrm{D}$ or $\mathcal{F}(t) \sim \rho(t) V(t)$ in $3 \mathrm{D}$, to determine $\rho(t)$ and $V(t)$ independently for each needle in the dendritic network. This approach rigorously bridges the dynamics of the dendrite tip and diffusion scales.

Sidebranches are generated periodically on the side of each dendrite, including fluctuations to represent the selective amplification of thermal noise at the origin of dendritic sidebranching [138]. The time evolution of $\rho V^{2}$ (in 2D) or $\rho V$ (in 3D) as a function of the flux intensity factor $\mathcal{F}(t)$ captures the evolution of the solute field surrounding each dendrite tip and the resulting evolution of $V(t)$, hence making it possible to model branches interactions and their unsteady growth competition.

The DNN model reproduces the Ivantsov steady-state solutions for a single free dendrite in 2D and 3D [140], as well as analytical laws for early-stage transient growth of idealized crossshaped equiaxed crystals in 2D [138] and 3D [140]. (The sharp needle formulation in [138] actually reproduces an approximation of the Ivantsov solution at low supersaturation.) The three-dimensional DNN model applied to unsteady competitive sidebranch growth [137] yields a good agreement with experimentally measured scaling laws for dendrite envelopes [88, 94]. First applications to directional solidification experiments show an excellent agreement on primary spacing selection [140]. For instance, in Fig. 6, the directional solidification of an Al12at. $\% \mathrm{Cu}$ alloy at a temperature gradient $G=62 \mathrm{~K} / \mathrm{cm}$ and a growth velocity $V=115 \mu \mathrm{m} / \mathrm{s}$ yields an average measured spacing $\Lambda_{\text {exp. }} \approx 258 \mu \mathrm{m}$, while DNN simulations predict an average spacing $\Lambda_{3 \mathrm{D}} \approx 237 \mu \mathrm{m}$.
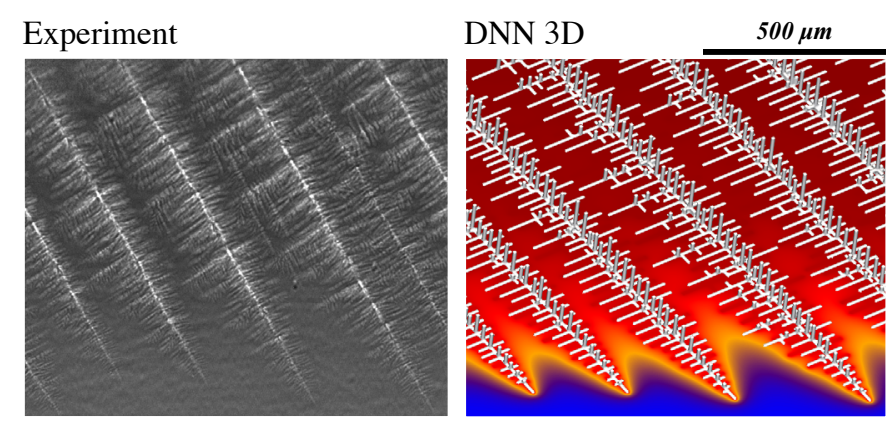

Figure 6: Spacing selection during directional solidification of an $\mathrm{Al}-12 \mathrm{at} . \% \mathrm{Cu}$ alloy at $G=62 \mathrm{~K} / \mathrm{cm}$ and $V=115 \mu \mathrm{m} / \mathrm{s}$ in the direction of the crystal growth (tilted by $30^{\circ}$ with respect to gravity and thermal gradient). The $\mathrm{X}$-ray image in (a) and the 3D simulation in (b) are represented at the same scale. [140]

\section{Outlook}

The last two decades have witnessed major progress in atomistic simulations methods to compute key interfacial properties impacting solidification microstructures. However, quantitative predictions of those properties remain scarce for alloys. Atomistic modeling is needed to predict interfacial energy anisotropies in the Al-Zn system in order to link more directly continuum scale modeling to experimentally observed changes of dendrite growth directions as a function of $\mathrm{Zn}$ concentration. Current atomistic modeling predictions also fall short explaining the full spectrum of dendrite growth directions and morphologies observed in rapid alloy solidification [27]. In particular, existing atomistic computations of interface kinetic anisotropy predict that dendrite growth along $\langle 100\rangle$ directions should be favored at large growth rate while other directions $\langle 111\rangle$, and disordered growth modes that may underly grain refinement, are also observed. In addition, atomistic modeling studies of interface coalescence, which have so far focused on pure metals, remain to be extended to binary alloys. The results of such studies, used in conjunction with mesoscale modeling, could potentially help explain how hot cracking sensitivity depends on the choice and concentration of alloying elements. The increased availability of EAM interatomic potentials for different alloy systems should facilitate such studies.

At the continuum scale, PF modeling has been used successfully to model a wide range of solidification microstructures, with the last few years witnessing the most rapid advances to simulate experimentally relevant length and time scales in 3D. However, despite advances in computing technologies, quantitative PF modeling still relies on formulating models with the desired thin-interface limit. While there has been significant recent advances in formulating new PF models for a wider range of transport properties, as well as concentrated and multicomponent alloys, extensions of those models and more extensive benchmarking of their predictions remain needed.

At the grain scale, multiscale approaches like the dendritic needle network model should pave the way to quantitative studies of dendritic microstructure selection mechanisms at length and time scales out of the reach of phase-field models. Large scale simulations of dendritic structures should yield significant 
insight into complex mechanisms such as grain growth competition [139], the long range effect of fluid flow on microstructure selection [126], and the columnar-to-equiaxed transition [165].

\section{Acknowledgements}

A.K. acknowledges support of grant DEFG02-07ER46400 from the US Department of Energy, Office of Basic Energy Sciences, for support of atomistic-scale phase-field-crystal modeling of interfacial properties, and NASA for continuumscale phase-field and dendritic-needle-network modeling of cellular/dendritic microstructures. D.T. is supported by Amy Clarke's Early Career award from the U.S. DOE, Office of Basic Energy Sciences, Division of Materials Sciences and Engineering and Los Alamos National Laboratory, operated by Los Alamos National Security, LLC under Contract No. DE-AC5206NA25396 for the U.S. Department of Energy.

\section{References}

[1] Adland, A., Karma, A., Spatschek, R., Buta, D., Asta, M., 2013. Phasefield-crystal study of grain boundary premelting and shearing in bcc iron. Physical Review B 87 (2), 024110.

[2] Ahmad, N., Wheeler, A., Boettinger, W., McFadden, G., 1998. Solute trapping and solute drag in a phase-field model of rapid solidification. Physical Review E 58 (3), 3436.

[3] Amoorezaei, M., Gurevich, S., Provatas, N., 2010. Spacing characterization in Al-Cu alloys directionally solidified under transient growth conditions. Acta Materialia 58 (18), 6115-6124.

[4] Amoorezaei, M., Gurevich, S., Provatas, N., 2012. Orientation selection in solidification patterning. Acta Materialia 60 (2), 657-663.

[5] Asta, M., Beckermann, C., Karma, A., Kurz, W., Napolitano, R., Plapp, M., Purdy, G., Rappaz, M., Trivedi, R., 2009. Solidification microstructures and solid-state parallels: Recent developments, future directions. Acta Materialia 57 (4), 941-971.

[6] Athreya, B. P., Goldenfeld, N., Dantzig, J. A., Greenwood, M., Provatas, N., 2007. Adaptive mesh computation of polycrystalline pattern formation using a renormalization-group reduction of the phase-field crystal model. Physical Review E 76 (5), 056706.

[7] Aziz, M. J., Kaplan, T., 1988. Continuous growth model for interface motion during alloy solidification. Acta Metallurgica 36 (8), 2335-2347.

[8] Balluffi, R., Maurer, R., 1988. On rotating sphere-on-a-plate experiments and the question of whether high angle grain boundaries melt below bulk melting temperatures. Scripta Metallurgica 22 (5), 709-713.

[9] Barbieri, A., Langer, J., 1989. Predictions of dendritic growth rates in the linearized solvability theory. Physical Review A 39 (10), 5314

[10] Becker, C., Olmsted, D., Asta, M., Hoyt, J., Foiles, S., 2007. Atomistic underpinnings for orientation selection in alloy dendritic growth. Physical Review Letters 98 (12), 125701.

[11] Beckermann, C., Diepers, H.-J., Steinbach, I., Karma, A., Tong, X., 1999. Modeling melt convection in phase-field simulations of solidification. Journal of Computational Physics 154 (2), 468-496.

[12] Bergeon, N., Tourret, D., Chen, L., Debierre, J.-M., Guérin, R., Ramirez, A., Billia, B., Karma, A., Trivedi, R., 2013. Spatiotemporal dynamics of oscillatory cellular patterns in three-dimensional directional solidification. Physical Review Letters 110 (22), 226102.

[13] Berghoff, M., Selzer, M., Choudhury, A., Nestler, B., ???? Efficient techniques for bridging from atomic to mesoscopic scale in phase-field simulations. Journal of Computational Methods in Sciences and Engineering.

[14] Berry, J., Elder, K., Grant, M., 2008. Melting at dislocations and grain boundaries: A phase field crystal study. Physical Review B 77 (22), 224114.

[15] Boettinger, W., Coriell, S., Greer, A., Karma, A., Kurz, W., Rappaz, M., Trivedi, R., 2000. Solidification microstructures: recent developments, future directions. Acta materialia 48 (1), 43-70.
[16] Boettinger, W., Warren, J., Beckermann, C., Karma, A., 2002. Phasefield simulation of solidification. Annual Review of Materials Research 32 (1), 163-194.

[17] Bollada, P., Goodyer, C., Jimack, P., Mullis, A., Yang, F., 2015. Three dimensional thermal-solute phase field simulation of binary alloy solidification. Journal of Computational Physics 287, 130-150.

[18] Böttger, B., Eiken, J., Apel, M., 2009. Phase-field simulation of microstructure formation in technical castings-a self-consistent homoenthalpic approach to the micro-macro problem. Journal of Computational Physics 228 (18), 6784-6795.

[19] Boussinot, G., Brener, E. A., 2014. Achieving realistic interface kinetics in phase-field models with a diffusional contrast. Physical Review E 89, 060402.

[20] Boussinot, G., Brener, E. A., Hueter, C., Spatschek, R., 2014. Elimination of surface diffusion in the non-diagonal phase field model. arXiv preprint arXiv: 1411.0523.

[21] Bragard, J., Karma, A., Lee, Y. H., Plapp, M., 2002. Linking phasefield and atomistic simulations to model dendritic solidification in highly undercooled melts. Interface Science 10 (2-3), 121-136.

[22] Brener, E., Mel'Nikov, V., 1991. Pattern selection in two-dimensional dendritic growth. Advances in Physics 40 (1), 53-97.

[23] Brener, E. A., Boussinot, G., 2012. Kinetic cross coupling between nonconserved and conserved fields in phase field models. Physical Review E 86 (6), 060601.

[24] Broughton, J., Gilmer, G., 1986. Thermodynamic criteria for grainboundary melting: a molecular-dynamics study. Physical Review Letters $56(25), 2692$.

[25] Cahn, J. W., Mishin, Y., Suzuki, A., 2006. Coupling grain boundary motion to shear deformation. Acta Materialia 54 (19), 4953-4975.

[26] Carozzani, T., Gandin, C.-A., Digonnet, H., Bellet, M., Zaidat, K., Fautrelle, Y., 2013. Direct simulation of a solidification benchmark experiment. Metallurgical and Materials Transactions A 44 (2), 873-887.

[27] Castle, E. G., Mullis, A. M., Cochrane, R. F., 2014. Evidence for an extended transition in growth orientation and novel dendritic seaweed structures in undercooled cu-8.9 wt\% ni. Journal of Alloys and Compounds 615, S612-S615.

[28] Chan, S.-W., Liu, J., Balluffi, R., 1985. Test for a possible "melting" transition in grain boundaries in aluminum near the melting point. Scripta Metallurgica 19 (10), 1251-1255.

[29] Chen, L.-Q., 2002. Phase-field models for microstructure evolution. Annual Review of Materials Research 32 (1), 113-140.

[30] Chernov, A., 2004. Notes on interface growth kinetics 50 years after Burton, Cabrera and Frank. Journal of Crystal Growth 264 (4), 499-518.

[31] Choudhury, A., Nestler, B., 2012. Grand-potential formulation for multicomponent phase transformations combined with thin-interface asymptotics of the double-obstacle potential. Physical Review E 85 (2), 021602.

[32] Clarke, D. R., 1987. On the equilibrium thickness of intergranular glass phases in ceramic materials. Journal of the American Ceramic Society 70 (1), 15-22.

[33] Couder, Y., Maurer, J., González-Cinca, R., Hernández-Machado, A., 2005. Side-branch growth in two-dimensional dendrites. I. experiments. Physical Review E 71 (3), 031602.

[34] Danilov, D. A., Lebedev, V. G., Galenko, P. K., 2014. A grand potential approach to phase-field modeling of rapid solidification. Journal of NonEquilibrium Thermodynamics 39 (2), 93-111.

[35] Dantzig, J., Di Napoli, P., Friedli, J., Rappaz, M., 2013. Dendritic growth morphologies in Al-Zn alloys - Part II: Phase-field computations. Metallurgical and Materials Transactions A 44 (12), 5532-5543.

[36] Dash, J., Fu, H., Wettlaufer, J., 1995. The premelting of ice and its environmental consequences. Reports on Progress in Physics 58 (1), 115.

[37] Divinski, S., Lohmann, M., Herzig, C., Straumal, B., Baretzky, B., Gust, W., 2005. Grain-boundary melting phase transition in the $\mathrm{Cu}-\mathrm{Bi}$ system. Physical Review B 71 (10), 104104.

[38] Echebarria, B., Folch, R., Karma, A., Plapp, M., 2004. Quantitative phase-field model of alloy solidification. Physical Review E 70 (6), 061604.

[39] Echebarria, B., Karma, A., Gurevich, S., 2010. Onset of sidebranching in directional solidification. Physical Review E 81 (2), 021608.

[40] Eiken, J., 2008. Dendritic orientation selection and growth texture evolution during directional solidification of magnesium-based alloys in- 
vestigated by phase- field simulations. Second International Conference on Advances in Solidification Processes (ICASP-2), June 17-20, 2008, University of Leoben, Austria.

[41] Eiken, J., 2009. Dendritic growth texture evolution in Mg-based alloys investigated by phase-field simulation. International Journal of Cast Metals Research 22 (1-4), 86-89.

[42] Eiken, J., Böttger, B., Steinbach, I., 2006. Multiphase-field approach for multicomponent alloys with extrapolation scheme for numerical application. Physical Review E 73 (6), 066122.

[43] Emmerich, H., 2003. The diffuse interface approach in materials science: thermodynamic concepts and applications of phase-field models. Vol. 73. Springer Science \& Business Media.

[44] Fallah, V., Amoorezaei, M., Provatas, N., Corbin, S., Khajepour, A., 2012. Phase-field simulation of solidification morphology in laser powder deposition of Ti-Nb alloys. Acta Materialia 60 (4), 1633-1646.

[45] Fattebert, J.-L., Wickett, M., Turchi, P., 2014. Phase-field modeling of coring during solidification of au-ni alloy using quaternions and calphad input. Acta Materialia 62, 89-104.

[46] Fensin, S. J., Olmsted, D., Buta, D., Asta, M., Karma, A., Hoyt, J., 2010. Structural disjoining potential for grain-boundary premelting and grain coalescence from molecular-dynamics simulations. Physical Review E 81 (3), 031601.

[47] Friedli, J., Fife, J., Di Napoli, P., Rappaz, M., 2013. Dendritic growth morphologies in Al-Zn alloys - Part I: X-ray tomographic microscopy. Metallurgical and Materials Transactions A 44 (12), 5522-5531.

[48] Frolov, T., Mishin, Y., 2009. Solid-liquid interface free energy in binary systems: Theory and atomistic calculations for the (110) CuAg interface. J. Chem. Phys. 131, 054702.

[49] Galenko, P., Abramova, E., Jou, D., Danilov, D., Lebedev, V., Herlach, D., 2011. Solute trapping in rapid solidification of a binary dilute system: A phase-field study. Physical Review E 84 (4), 041143.

[50] Galenko, P., Sobolev, S., 1997. Local nonequilibrium effect on undercooling in rapid solidification of alloys. Physical Review E 55 (1), 343.

[51] Gandin, C.-A., 2010. Modeling of solidification: Grain structures and segregations in metallic alloys. Comptes Rendus Physique 11 (3), 216225

[52] Gandin, C.-A., Eshelman, M., Trivedi, R., 1996. Orientation dependence of primary dendrite spacing. Metallurgical and Materials Transactions A 27 (9), 2727-2739.

[53] Gao, Y. F., Yang, Y., Sun, D. Y., Asta, M., Hoyt, J. J., 2010. Molecular dynamics simulations of the crystal-melt interface mobility in hcp $\mathrm{Mg}$ and bcc Fe. Journal of Crystal Growth 312 (21), 3238-3242.

[54] George, W. L., Warren, J. A., 2002. A parallel 3d dendritic growth simulator using the phase-field method. Journal of Computational Physics 177 (2), 264-283

[55] Georgelin, M., Pocheau, A., 1997. Oscillatory instability, limit cycle, and transition to doublets in directional solidification. Physical Review Letters 79 (14), 2698.

[56] Ghmadh, J., Debierre, J.-M., Deschamps, J., Georgelin, M., Guérin, R., Pocheau, A., 2014. Directional solidification of inclined structures in thin samples. Acta Materialia 74, 255-267.

[57] Gruber, J., Ma, N., Wang, Y., Rollett, A., Rohrer, G., 2006. Sparse data structure and algorithm for the phase field method. Modelling and simulation in materials science and engineering 14 (7), 1189

[58] Guo, Z., Mi, J., Grant, P., 2012. Phase field simulation of multi-dendrite growth in a coupled thermal-solute-convective environment. In: IOP Conference Series: Materials Science and Engineering. Vol. 33. IOP Publishing, p. 012101

[59] Gupta, V. K., Yoon, D.-H., Meyer, H. M., Luo, J., 2007. Thin intergranular films and solid-state activated sintering in nickel-doped tungsten. Acta Materialia 55 (9), 3131-3142.

[60] Gurevich, S., Amoorezaei, M., Montiel, D., Provatas, N., 2012. Evolution of microstructural length scales during solidification of magnesium alloys. Acta Materialia 60 (8), 3287-3295.

[61] Gurevich, S., Amoorezaei, M., Provatas, N., 2010. Phase-field study of spacing evolution during transient growth. Physical Review E 82 (5), 051606.

[62] Gurevich, S., Karma, A., Plapp, M., Trivedi, R., 2010. Phase-field study of three-dimensional steady-state growth shapes in directional solidification. Physical Review E 81 (1), 011603.

[63] Haxhimali, T., Karma, A., Gonzales, F., Rappaz, M., 2006. Orientation selection in dendritic evolution. Nature Materials 5 (8), 660-664.

[64] Henry, H., Mellenthin, J., Plapp, M., 2012. Orientation-field model for polycrystalline solidification with a singular coupling between order and orientation. Physical Review B 86 (5), 054117.

[65] Hoyt, J., Asta, M., Karma, A., 2001. Method for computing the anisotropy of the solid-liquid interfacial free energy. Physical Review Letters 86 (24), 5530

[66] Hoyt, J., Asta, M., Karma, A., 2003. Atomistic and continuum modeling of dendritic solidification. Materials Science and Engineering: R: Reports 41 (6), 121-163.

[67] Hoyt, J., Olmsted, D., Jindal, S., Asta, M., Karma, A., 2009. Method for computing short-range forces between solid-liquid interfaces driving grain boundary premelting. Physical Review E 79 (2), 020601.

[68] Hoyt, J., Sadigh, B., Asta, M., Foiles, S., 1999. Kinetic phase field parameters for the cu-ni system derived from atomistic computations. Acta Materialia 47 (11), 3181-3187.

[69] Hoyt, J. J., Asta, M., Karma, A., 2002. Atomistic simulation methods for computing the kinetic coefficient in solid-liquid systems. Interface Science 10 (2-3), 181-189.

[70] Hoyt, J. J., Asta, M., Karma, A., 2012. Atomistic simulations of solute trapping and solute drag. In: Herlach, D. M., Matson, D. M. (Eds.), Solidification of Containerless Undercooled Melts. Wiley, pp. 363-380.

[71] Hsieh, T., Balluffi, R., 1989. Observations of roughening/de-faceting phase transitions in grain boundaries. Acta Metallurgica 37 (8), 2133 2139.

[72] Ihle, T., 2000. Competition between kinetic and surface tension anisotropy in dendritic growth. The European Physical Journal BCondensed Matter and Complex Systems 16 (2), 337-344.

[73] Inoko, F., Okada, T., Muraga, T., Nakano, Y., Yoshikawa, T., 1997. Strain induced grain boundary premelting in bulk copper bicrystals. Interface Science 4 (3-4), 263-272.

[74] Ivanov, V., Mishin, Y., 2008. Dynamics of grain boundary motion coupled to shear deformation: An analytical model and its verification by molecular dynamics. Physical Review B 78 (6), 064106.

[75] Jackson, K. A., Beatty, K. M., Gudgel, K. A., 2004. An analytical model for non-equilibrium segregation during crystallization. Journal of crystal growth 271 (3), 481-494.

[76] Karma, A., 2001. Dendritic growth. In: Fleury, V., Gouyet, J. F., Lonetti, M. (Eds.), Branching in Nature. Springer, pp. 365-402.

[77] Karma, A., 2001. Phase-field formulation for quantitative modeling of alloy solidification. Physical Review Letters 87 (11), 115701.

[78] Karma, A., Rappel, W.-J., 1996. Numerical simulation of threedimensional dendritic growth. Physical Review Letters 77 (19), 4050.

[79] Karma, A., Rappel, W.-J., 1998. Quantitative phase-field modeling of dendritic growth in two and three dimensions. Physical review E 57 (4), 4323.

[80] Karma, A., Trautt, Z. T., Mishin, Y., 2012. Relationship between equilibrium fluctuations and shear-coupled motion of grain boundaries. Physical Review Letters 109 (9), 095501

[81] Kessler, D. A., Koplik, J., Levine, H., 1988. Pattern selection in fingered growth phenomena. Advances in Physics 37 (3), 255-339.

[82] Kikuchi, R., Cahn, J. W., 1980. Grain-boundary melting transition in a two-dimensional lattice-gas model. Physical Review B 21 (5), 1893.

[83] Kobayashi, R., Warren, J. A., Carter, W. C., 2000. A continuum model of grain boundaries. Physica D: Nonlinear Phenomena 140 (1), 141-150.

[84] Kurtuldu, G., Jarry, P., Rappaz, M., 2013. Influence of cr on the nucleation of primary al and formation of twinned dendrites in al-zn-cr alloys: Can icosahedral solid clusters play a role? Acta Materialia 61 (19), 7098-7108.

[85] Kurtuldu, G., Sicco, A., Rappaz, M., 2014. Icosahedral quasicrystalenhanced nucleation of the fcc phase in liquid gold alloys. Acta Materialia 70, 240-248.

[86] Langer, J. S., 1987. Chance and Matter, Lectures on the Theory of Pastern Formation, Les Houches, Session XLVI. North-Holland, Amsterdam, pp. 629-711.

[87] Li, J., Wang, Z., Wang, Y., Wang, J., 2012. Phase-field study of competitive dendritic growth of converging grains during directional solidification. Acta Materialia 60 (4), 1478-1493.

[88] Li, Q., Beckermann, C., 1999. Evolution of the sidebranch structure in free dendritic growth. Acta materialia 47 (8), 2345-2356.

[89] Lobkovsky, A. E., Warren, J. A., 2002. Phase field model of premelting 
of grain boundaries. Physica D: Nonlinear Phenomena 164 (3), 202-212.

[90] Losert, W., Shi, B., Cummins, H., Warren, J., 1996. Spatial perioddoubling instability of dendritic arrays in directional solidification. Physical Review Letters 77 (5), 889.

[91] Luo, J., Gupta, V., Yoon, D., Meyer III, H., 2005. Segregation-induced grain boundary premelting in nickel-doped tungsten. Applied Physics Letters 87 (23), 231902.

[92] Masumura, R., Glicksman, M., Vold, C., 1972. Absolute solid-liquid and grain boundary energies of bismuth. Scripta Metallurgica 6 (10), 943-946.

[93] Medvedev, D., Kassner, K., 2005. Lattice Boltzmann scheme for crystal growth in external flows. Physical Review E 72 (5), 056703.

[94] Melendez, A., Beckermann, C., 2012. Measurements of dendrite tip growth and sidebranching in succinonitrile-acetone alloys. Journal of Crystal Growth 340 (1), 175-189.

[95] Mellenthin, J., Karma, A., Plapp, M., 2008. Phase-field crystal study of grain-boundary premelting. Physical Review B 78 (18), 184110.

[96] Mendelev, M., Rahman, M., Hoyt, J., Asta, M., 2010. Moleculardynamics study of solid-liquid interface migration in fcc metals. Modelling and Simulation in Materials Science and Engineering 18 (7), 074002 .

[97] Mikheev, L., Chernov, A., 1991. Mobility of a diffuse simple crystalmelt interface. Journal of Crystal Growth 112 (2), 591-596.

[98] Miller, W., Succi, S., Mansutti, D., 2001. Lattice Boltzmann model for anisotropic liquid-solid phase transition. Physical Review Letters 86 (16), 3578

[99] Mishin, Y., Boettinger, W., Warren, J., McFadden, G., 2009. Thermodynamics of grain boundary premelting in alloys. i. phase-field modeling. Acta Materialia 57 (13), 3771-3785.

[100] Moelans, N., 2011. A quantitative and thermodynamically consistent phase-field interpolation function for multi-phase systems. Acta Materialia 59 (3), 1077-1086

[101] Monk, J., Yang, Y., Mendelev, M. I., Asta, M., Hoyt, J. J., Sun, D. Y., 2010. Determination of the crystal-melt interface kinetic coefficient from molecular dynamics simulations. Modelling and Simulation in Materials Science and Engineering 18 (1), 015004

[102] Montiel, D., Gurevich, S., Ofori-Opoku, N., Provatas, N., 2014. Characterization of late-stage equiaxed solidification of alloys. Acta Materialia $77,183-190$.

[103] Montiel, D., Liu, L., Xiao, L., Zhou, Y., Provatas, N., 2012. Microstructure analysis of az31 magnesium alloy welds using phase-field models. Acta Materialia 60 (16), 5925-5932.

[104] Mullis, A., 2011. Prediction of the operating point of dendrites growing under coupled thermosolutal control at high growth velocity. Physical Review E 83 (6), 061601.

[105] Mullis, A., Rosam, J., Jimack, P., 2010. Solute trapping and the effects of anti-trapping currents on phase-field models of coupled thermo-solutal solidification. Journal of Crystal Growth 312 (11), 1891-1897.

[106] Nestler, B., 2005. A 3d parallel simulator for crystal growth and solidification in complex alloy systems. Journal of Crystal Growth 275 (1), e273-e278.

[107] Nestler, B., Aksi, A., Selzer, M., 2010. Combined lattice Boltzmann and phase-field simulations for incompressible fluid flow in porous media. Mathematics and Computers in Simulation 80 (7), 1458-1468.

[108] Neumann-Heyme, H., Eckert, K., Voigt, A., Odenbach, S., 2012. Growth of a free dendrite in pure substances under modulated flow conditions. In: IOP Conference Series: Materials Science and Engineering. Vol. 33. IOP Publishing, p. 012106

[109] Ofori-Opoku, N., Provatas, N., 2010. A quantitative multi-phase field model of polycrystalline alloy solidification. Acta Materialia 58 (6), 2155-2164.

[110] Ohno, M., 2012. Quantitative phase-field modeling of nonisothermal solidification in dilute multicomponent alloys with arbitrary diffusivities. Physical Review E 86 (5), 051603.

[111] Olmsted, D. L., Buta, D., Adland, A., Foiles, S. M., Asta, M., Karma, A., 2011. Dislocation-pairing transitions in hot grain boundaries. Physical Review Letters 106 (4), 046101.

[112] Plapp, M., 2011. Remarks on some open problems in phase-field modelling of solidification. Philosophical Magazine 91 (1), 25-44.

[113] Plapp, M., 2011. Unified derivation of phase-field models for alloy solidification from a grand-potential functional. Physical Review E 84 (3),
031601

[114] Plapp, M., 2015. Current Opinion in Solid State \& Materials Science this issue.

[115] Plapp, M., Karma, A., 2000. Multiscale random-walk algorithm for simulating interfacial pattern formation. Physical Review Letters 84 (8), 1740.

[116] Provatas, N., Goldenfeld, N., Dantzig, J., 1998. Efficient computation of dendritic microstructures using adaptive mesh refinement. Physical Review Letters 80 (15), 3308.

[117] Provatas, N., Greenwood, M., Athreya, B., Goldenfeld, N., Dantzig, J., 2005. Multiscale modeling of solidification: phase-field methods to adaptive mesh refinement. International Journal of Modern Physics B 19 (31), 4525-4565.

[118] Rappaz, M., 2015. Modeling and characterization of grain structures and defects in solidification. Current Opinion in Solid State \& Materials Science this issue.

[119] Rappaz, M., Gandin, C.-A., 1993. Probabilistic modelling of microstructure formation in solidification processes. Acta Metallurgica et materialia 41 (2), 345-360.

[120] Rappaz, M., Jacot, A., Boettinger, W., 2003. Last-stage solidification of alloys: Theoretical model of dendrite-arm and grain coalescence. Metallurgical and Materials Transactions A 34 (3), 467-479.

[121] Sai Pavan Kumar Bhogireddy, V., Hüter, C., Neugebauer, J., Steinbach, I., Karma, A., Spatschek, R., 2014. Phase-field modeling of grain-boundary premelting using obstacle potentials. Physical Review E 90 (1), 012401.

[122] Salgado-Ordorica, M., Burdet, P., Cantoni, M., Rappaz, M., 2011. Study of the twinned dendrite tip shape ii: Experimental assessment. Acta Materialia 59 (13), 5085-5091.

[123] Salgado-Ordorica, M., Desbiolles, J.-L., Rappaz, M., 2011. Study of the twinned dendrite tip shape i: Phase-field modeling. Acta Materialia 59 (13), 5074-5084.

[124] Shimokawabe, T., Aoki, T., Takaki, T., Yamanaka, A., Nukada, A., Endo, T., Maruyama, N., Matsuoka, S., 2011. Peta-scale phase-field simulation for dendritic solidification on the TSUBAME 2.0 supercomputer. In: High Performance Computing, Networking, Storage and Analysis (SC), 2011 International Conference for. IEEE, pp. 1-11.

[125] Song, Y., Karma, A., 2015. Unpublished work.

[126] Steinbach, I., 2009. Pattern formation in constrained dendritic growth with solutal buoyancy. Acta Materialia 57 (9), 2640-2645.

[127] Steinbach, I., Pezzolla, F., Nestler, B., Seeßelberg, M., Prieler, R., Schmitz, G., Rezende, J., 1996. A phase field concept for multiphase systems. Physica D: Nonlinear Phenomena 94 (3), 135-147.

[128] Steinbach, I., Zhang, L., Plapp, M., 2012. Phase-field model with finite interface dissipation. Acta Materialia 60 (6), 2689-2701.

[129] Sun, D., Zhu, M., Pan, S., Raabe, D., 2009. Lattice Boltzmann modeling of dendritic growth in a forced melt convection. Acta Materialia 57 (6), $1755-1767$.

[130] Sun, D. Y., Asta, M., Hoyt, J. J., 2004. Crystal-melt interfacial free energies and mobilities in fcc and bcc Fe. Physical Review B 69 (17), 174103

[131] Sun, Y., Beckermann, C., 2009. Effect of solid-liquid density change on dendrite tip velocity and shape selection. Journal of Crystal Growth 311 (19), 4447-4453.

[132] Takaki, T., Ohno, M., Shimokawabe, T., Aoki, T., 2014. Twodimensional phase-field simulations of dendrite competitive growth during the directional solidification of a binary alloy bicrystal. Acta Materialia $81,272-283$.

[133] Takaki, T., Shimokawabe, T., Ohno, M., Yamanaka, A., Aoki, T., 2013. Unexpected selection of growing dendrites by very-large-scale phasefield simulation. Journal of Crystal Growth 382, 21-25.

[134] Tang, M., Carter, W. C., Cannon, R. M., 2006. Diffuse interface model for structural transitions of grain boundaries. Physical Review B 73 (2), 024102 .

[135] Tang, M., Carter, W. C., Cannon, R. M., 2006. Grain boundary transitions in binary alloys. Physical Review Letters 97 (7), 075502.

[136] Tourret, D., 2015. Unpublished work.

[137] Tourret, D., Karma, 2015. Three-dimensional dendritic needle network model for alloy solidification. Article in preparation.

[138] Tourret, D., Karma, A., 2013. Multiscale dendritic needle network model of alloy solidification. Acta Materialia 61 (17), 6474-6491. 
[139] Tourret, D., Karma, A., 2015. Growth competition of columnar dendritic grains: A phase-field study. Acta Materialia 82, 64-83.

[140] Tourret, D., Karma, A., Clarke, A., Gibbs, P., Imhoff, S., 2015. Threedimensional Dendritic Needle Network model with application to Al-Cu directional solidification experiments. IOP Conference Series: Materials Science and Engineering 84 (1), 012082.

[141] Trautt, Z., Adland, A., Karma, A., Mishin, Y., 2012. Coupled motion of asymmetrical tilt grain boundaries: Molecular dynamics and phase field crystal simulations. Acta Materialia 60 (19), 6528-6546.

[142] Trautt, Z., Mishin, Y., 2012. Grain boundary migration and grain rotation studied by molecular dynamics. Acta Materialia 60 (5), 2407-2424.

[143] Turnbull, D., 1950. Formation of crystal nuclei in liquid metals. Journal of Applied Physics 21 (10), 1022-1028.

[144] Vanherpe, L., Moelans, N., Blanpain, B., Vandewalle, S., 2007. Bounding box algorithm for three-dimensional phase-field simulations of microstructural evolution in polycrystalline materials. Physical Review E 76 (5), 056702.

[145] Vold, C., Glicksman, M., 1972. Behavior of grain boundaries near the melting point. In: The Nature and Behavior of Grain Boundaries. Metallurgical Society of AIME, Plenum Press, New York, pp. 171-183.

[146] Von Alfthan, S., Kaski, K., Sutton, A., 2007. Molecular dynamics simulations of temperature-induced structural transitions at twist boundaries in silicon. Physical Review B 76 (24), 245317.

[147] Walton, D., Chalmers, B., 1959. The origin of the preferred orientation in the columnar zone of ingots. Transactions of the American Institute of Mining and Metallurgical Engineers 215 (3), 447-457.

[148] Wang, H., Kuang, W., Zhang, X., Liu, F., 2015. A hyperbolic phasefield model for rapid solidification of a binary alloy. Journal of Materials Science 50 (3), 1277-1286.

[149] Wang, H., Liu, F., Ehlen, G., Herlach, D., 2013. Application of the maximal entropy production principle to rapid solidification: A multi-phasefield model. Acta Materialia 61 (7), 2617-2627.

[150] Wang, N., Mokadem, S., Rappaz, M., Kurz, W., 2004. Solidification cracking of superalloy single-and bi-crystals. Acta Materialia 52 (11), 3173-3182.

[151] Wang, N., Spatschek, R., Karma, A., 2010. Multi-phase-field analysis of short-range forces between diffuse interfaces. Physical Review E 81 (5), 051601.

[152] Watanabe, T., Kimura, S.-I., Karashima, S., 1984. The effect of a grain boundary structural transformation on sliding in ${ }_{i} 1010_{i}$-tilt zinc bicrystals. Philosophical Magazine A 49 (6), 845-864.

[153] Widom, B., 1978. Structure of the $\alpha \gamma$ interface. The Journal of Chemical Physics 68 (8), 3878-3883.

[154] Williams, P., Mishin, Y., 2009. Thermodynamics of grain boundary premelting in alloys. II. Atomistic simulation. Acta Materialia 57 (13), 3786-3794.

[155] Wu, K.-A., Karma, A., Hoyt, J. J., Asta, M., 2006. Ginzburg-Landau theory of crystalline anisotropy for bcc-liquid interfaces. Physical Review B 73 (9), 094101.

[156] Wu, K.-A., Wang, C.-H., Hoyt, J. J., Karma, A., 2015. Ginzburg-Landau theory of the bcc-liquid interface kinetic coefficient. Physical Review B 91 (1), 014107.

[157] Yamanaka, A., Aoki, T., Ogawa, S., Takaki, T., 2011. GPU-accelerated phase-field simulation of dendritic solidification in a binary alloy. Journal of Crystal Growth 318 (1), 40-45.

[158] Yang, Y., Humadi, H., Buta, D., Laird, B. B., Sun, D., Hoyt, J. J., Asta, M., 2011. Atomistic simulations of nonequilibrium crystal-growth kinetics from alloy melts. Physical Review Letters 107 (2), 025505.

[159] Yin, H., Felicelli, S., Wang, L., 2011. Simulation of a dendritic microstructure with the lattice Boltzmann and cellular automaton methods. Acta Materialia 59 (8), 3124-3136.

[160] Yuan, L., Lee, P. D., 2010. Dendritic solidification under natural and forced convection in binary alloys: $2 \mathrm{D}$ versus 3D simulation. Modelling and Simulation in Materials Science and Engineering 18 (5), 055008.

[161] Zhang, L., Danilova, E. V., Steinbach, I., Medvedev, D., Galenko, P. K., 2013. Diffuse-interface modeling of solute trapping in rapid solidification: Predictions of the hyperbolic phase-field model and parabolic model with finite interface dissipation. Acta Materialia 61 (11), 41554168.

[162] Zhang, L., Steinbach, I., 2012. Phase-field model with finite interface dissipation: Extension to multi-component multi-phase alloys. Acta Ma- terialia 60 (6), 2702-2710.

[163] Zhang, L., Stratmann, M., Du, Y., Sundman, B., Steinbach, I., 2015. Incorporating the calphad sublattice approach of ordering into the phasefield model with finite interface dissipation. Acta Materialia 88, 156169.

[164] Zhou, Y., Volek, A., Green, N., 2008. Mechanism of competitive grain growth in directional solidification of a nickel-base superalloy. Acta Materialia 56 (11), 2631-2637.

[165] Zimmermann, G., Sturz, L., Billia, B., Mangelinck-Noël, N., Liu, D., Nguyen Thi, H., Bergeon, N., Gandin, C.-A., Browne, D., Beckermann, C., et al., 2014. Columnar-to-equiaxed transition in solidification processing of alsi7 alloys in microgravity the cetsol project. In: Materials Science Forum. Vol. 790. Trans Tech Publ, pp. 12-21. 